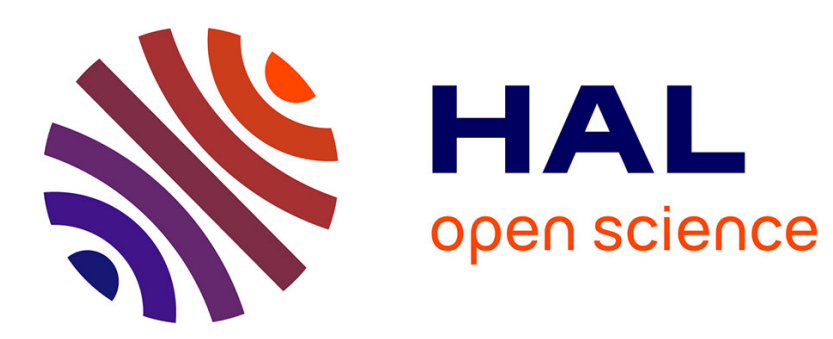

\title{
Sources de dépendance et stratégies pour innover. Une application aux studios de jeu vidéo français
}

\author{
Romain Gandia, Elodie Gardet
}

\section{To cite this version:}

Romain Gandia, Elodie Gardet. Sources de dépendance et stratégies pour innover. Une application aux studios de jeu vidéo français. Revue management \& avenir, 2012, 16 (56), pp.75-93. 10.3917/mav.056.0075 . hal-01293352

\section{HAL Id: hal-01293352 \\ https://hal.science/hal-01293352}

Submitted on 24 Mar 2016

HAL is a multi-disciplinary open access archive for the deposit and dissemination of scientific research documents, whether they are published or not. The documents may come from teaching and research institutions in France or abroad, or from public or private research centers.
L'archive ouverte pluridisciplinaire HAL, est destinée au dépôt et à la diffusion de documents scientifiques de niveau recherche, publiés ou non, émanant des établissements d'enseignement et de recherche français ou étrangers, des laboratoires publics ou privés. 


\title{
Sources de dépendance et stratégies pour innover. Une application aux studios de jeu vidéo français
}

\author{
Romain GANDIA \\ Doctorant en Sciences de Gestion \\ Université de Savoie (IREGE) \\ Elodie GARDET \\ Maître de Conférences en Sciences de Gestion \\ Université de Savoie (IREGE) \\ Institut de REcherche en Gestion et Economie (IREGE) \\ Université de Savoie - Domaine universitaire d'Annecy-le-Vieux \\ 4, Chemin de Bellevue - 74016 Annecy Cedex \\ Tél. : 0033 (0) 450092471 \\ Fax. : 0033 (0) 450092439
}

Pour toute correspondance : romain.gandia@univ-savoie.fr

\section{Résumé :}

Les entreprises ne maîtrisant pas l'intégralité du processus de création d'un produit sont sujettes à des situations de dépendance face à certains acteurs. La création d'un jeu vidéo nécessite l'intervention d'une multitude d'entreprises qui doivent se coordonner pour proposer un produit final attractif. Ces interactions conduisent à l'émergence d'une dépendance de certains acteurs au sein de la filière vis-à-vis d'autres. Les studios, situés au centre de cette filière, sont particulièrement intéressants à étudier car ils subissent une dépendance face aux acteurs tant de l'amont que de l'aval. L'objectif de cet article est d'apporter une contribution quant aux sources de dépendance influençant le choix stratégique: coopération ou intégration. En effet, les recherches existantes se focalisent essentiellement sur l'impact de ce choix sur la performance. Grâce à la réalisation de cinq études de cas, nous tentons d'examiner l'influence des différentes sources de dépendance sur le choix d'une stratégie de coopération ou d'intégration. Les résultats montrent que les stratégies mises en œuvre par les studios dépendent de la nature de la dépendance.

Mots clés : degré de dépendance, industrie culturelle, ressources et compétences. 


\section{INTRODUCTION}

Le développement d'un jeu vidéo implique la mobilisation d'une multitude d'acteurs au sein de la filière ${ }^{1}$, définie comme un ensemble articulé d'activités économiques exécutées par une suite d'intermédiaires (acteurs) au sein d'une industrie ou un réseau de relation (Toledano, 1978). Les fabricants créent la technologie et la plateforme de diffusion, les studios développent les jeux, les éditeurs financent et réalisent la promotion des jeux et les distributeurs alimentent les grossistes et détaillants. Les studios, situés au centre de cette filière, se retrouvent donc en situation de dépendance tant par rapport aux acteurs en amont (fabricants) qu'en aval (éditeurs et distributeurs). Cette dépendance permet eux acteurs dominant d'imposer leurs choix et de renforcer leur pouvoir (Blau, 1964). Ainsi, le pouvoir de A sur B représente la capacité de A d'obtenir que B fasse quelque chose qu'il n'aurait pas fait sans l'intervention de A (Dahl, 1957). De par cette dépendance, les studios ont une liberté créative limitée et ne capturent qu'une part réduite de la valeur créée (sous forme de royalties). Pour pallier ces effets négatifs, les studios tentent de minimiser leur degré de dépendance. Toutefois, dans la théorie de la dépendance des ressources (Pfeffer et Salancik, 1978), la dépendance est souvent considérée comme un facteur exogène sur lequel les entreprises n'ont pas d'influence (Gulati et Sytch, 2007). Ainsi, le «choix » stratégique d'une entreprise peut être subi et nous tentons ici de comprendre les sources qui sont à l'origine de ce choix.

Cette contribution identifie les sources de dépendance qui peuvent orienter le choix d'une stratégie ou d'une autre. Les deux principales stratégies sont l'intégration de ressources et compétences externes et le recours aux coopérations (Wang et Zajac, 2007). Les recherches analysant les avantages et inconvénients des coopérations ou des intégrations (Kogut et Singh, 1988 ; Hennart, 1988 ; Balakrishnan et Koza, 1993) apportent une compréhension limitée des sources motivant les entreprises à faire ce choix. Dans cet article, nous analysons la possible influence de la nature de la dépendance sur le choix stratégique. La problématique est la suivante : quelle est l'influence des sources de la dépendance sur les stratégies mises en œuvre par les entreprises?

La première partie de cette communication s'intéresse à l'identification des sources de dépendance à la fois internes et externes. Elle présente les stratégies que les entreprises peuvent instaurer pour tenter de minimiser cette dépendance. Le choix de l'industrie du jeu

\footnotetext{
${ }^{1}$ La filière est un outil de découpage structurel permettant de décrire la structure d'un système industriel (Toledano, 1978).
} 
vidéo, la méthode de recueil des données et les cinq cas étudiés sont détaillés dans une deuxième partie. La troisième partie présente les principaux résultats, discute du lien entre source de dépendance et stratégie mise en œuvre pour limiter la dépendance et conclut par des limites et perspectives de recherches futures.

\section{LOGIQUES DE DEPENDANCE}

Dans une filière, nécessitant l'intervention d'une multitude d'acteurs hétérogènes (métier, compétences, taille de l'entreprise, etc.), certains se retrouvent en situation de dépendance. Cette dépendance peut prendre la forme d'instructions négociées qui limite la liberté d'action des acteurs dépendants. Dans cette première partie, nous analysons les différentes sources de dépendance, puis les stratégies qui peuvent être mises en œuvre pour tenter de diminuer cette dépendance.

\subsection{ANALYSE INTERNE : LES RESSOURCES ET COMPETENCES DE L'ENTREPRISE}

$\mathrm{Au}$ sein de la filière, qui lie des entités interdépendantes conservant leur autonomie, la dépendance se réfère à la nécessité des entreprises de maintenir la relation avec tel ou tel acteur afin d'atteindre ses objectifs : la création d'une nouvelle technologie, la mise sur le marché d'un produit et la réalisation de bénéfices (Nooteboom et al., 2000). Le degré d'interdépendance détermine ainsi le degré de pouvoir de chacune des parties dans la relation, les deux étant inversement corrélés (Emerson, 1962).

Ressources et compétences : le manque de ressources et compétences est considéré, dans la littérature, comme une source majeure de dépendance (Lavie, 2006). En effet, l'entreprise est une entité composée de multiples ressources physiques (usines, équipements, terrains, matières premières, produits semi-finis, stocks) et humaines (travail qualifié ou non, personnel administratif, financier, juridique, technique, et de direction) qui doivent être durables et spécifiques pour obtenir un avantage concurrentiel. Les ressources sont dites spécifiques si elles sont immobiles et non transférables au travers de transactions commerciales (Conner et Prahalad, 1996). L'accent est mis sur la nécessité pour une entreprise de trouver des ressources (savoir-faire, technologies, hommes, etc.), de les acquérir, de les exploiter, mais aussi de savoir maitriser l'utilisation combinée de ressources internes et externes à des fins stratégiques (d'où les notions de compétences ou de capacités ${ }^{2}$ ). Pour qu'une compétence participe à la création d'un avantage concurrentiel durable (ce qui en fait

\footnotetext{
${ }^{2}$ De multiples définitions, que nous ne présentons pas ici, existent pour qualifier les notions de ressources, compétences ou capacités (parfois vues comme deux concepts distincts, parfois assimilées).
} 
une compétence « clé »), il faut qu'elle soit rare, qu'elle ne puisse pas être imitée ou achetée, et que son coût soit inférieur à la rente attendue. De multiples typologies identifient les types de ressources et compétences : l'objectif ici n'est pas d'en réaliser une catégorisation exhaustive mais d'analyser l'influence du type de ressources et compétences apportées par les acteurs d'une filière sur leur degré de dépendance.

Nous retenons ici les principales catégories de ressources (Arrègle, 2006) :

- Financières : disponibilité du capital ;

- Physiques: infrastructures productives, les investissements matériels et les moyens de distribution fournis par le membre ;

- Humaines : le personnel et les compétences que chacun possède ;

- Techniques : savoirs tacites ou liés à la capacité de développer un nouveau savoir-faire technologique permettant ainsi d'obtenir des droits de propriété via le dépôt de dessins et modèles ou brevets. La propriété intellectuelle (PI) est l'actif le plus important dans une entreprise (Chesbrough, 2003a). En la protégeant ${ }^{3}$, l'entreprise peut éviter les phénomènes d'imitation et les comportements opportunistes qui sont susceptibles de remettre en cause la survie ou la croissance de l'entreprise (Pisano et Teece, 2007). Cette protection permet également d'assurer l'appropriation effective des retours économiques liés à la PI (Chesbrough, 2003b). Les DPI, quant à eux, peuvent êtres considérés tant comme une nouvelle classe d'actifs que comme un outil de protection (Chesbrough et al., 2006). Ils peuvent servir d'actifs stratégiques (Corbel, 2003) car ils confèrent une valeur intrinsèque à l'entreprise. Celle-ci peut alors développer un avantage concurrentiel (Liotard, 1999) face à ses concurrents et augmenter son pouvoir de négociation ;

- Réputation de l'entreprise : associée à la fiabilité, elle est considérée comme un atout stratégique (Barney et Hansen, 1994) ${ }^{4}$. Parce que l'opportunisme est une préoccupation majeure dans les relations interorganisationnelles, la réputation d'une entreprise contribue à consolider sa position au sein d'une filière. Une «bonne » réputation permet de renforcer la confiance des autres acteurs de l'industrie (Das et Teng, 1998). Zaheer et al. (1998) constatent que la confiance interentreprises réduit les coûts de négociation et minimise les rapports de force. Par ailleurs, une entreprise qui possède une bonne réputation au sein de l'industrie trouvera plus facilement des fournisseurs et des distributeurs ;

\footnotetext{
${ }^{3}$ Ces protections peuvent prendre différentes formes : les brevets, les droits d'auteurs, etc. (Pisano et Teece, 2007).

${ }^{4}$ Dans cette recherche, dans la lignée de Das et Teng (1998), nous distinguons clairement la réputation des autres types de ressources. Toutefois, certaines recherches assimilent la réputation à une ressource intangible.
} 
Les ressources possédées en interne déterminent le niveau de dépendance d'un acteur par rapport aux autres - et donc son niveau de pouvoir (Blau, 1964). Ceci est d'autant plus important que l'analyse porte sur des relations asymétriques, complémentaires, dont la finalité est, pour les acteurs, l'accès à des ressources détenues par les autres membres (Hamel et Prahalad, 1994). Certaines caractéristiques des ressources tendent à renforcer (ou diminuer) le pouvoir de négociation d'un acteur : le niveau de spécificité des actifs engagés (Brousseau, 1993 ; Williamson, 1985), l'accès à la ressource, son niveau de concentration (Pfeffer et Salancik, 1978) et sa valeur intrinsèque. Ainsi, les différents acteurs de la filière doivent interagir avec d'autres entreprises qui possèdent des ressources et compétences complémentaires, ce qui risque d'engendrer des situations de dépendance (Arrègle, 2006).

Le manque de ressources et compétences en interne est la source la plus souvent citée pour expliquer la situation de dépendance (Lavie, 2006). Par ailleurs, la taille a également été identifiée comme une source possible de dépendance d'une entreprise. En effet, un acteur de taille importante dispose généralement d'un pouvoir supérieur au sein de la filière (Oliver, 1990). Les négociations entre une entreprise de taille réduite et une entreprise plus importante sont souvent en faveur de la plus grande entreprise. En effet, les interactions avec d'autres acteurs de la filière revêtent souvent pour la petite entreprise un caractère stratégique plus prononcé que pour la grande entreprise - d'où un déséquilibre initial en matière de pouvoir de négociation (Tinlot et Mothe, 2005).

\subsection{ANALYSE EXTERNE : LES AUTRES SOURCES DE DEPENDANCE}

Outre les sources internes de dépendance, deux principales sources externes peuvent être mises en avant au regard de la littérature : la nature de la technologie (le support) et la position au sein de la filière.

Nature de la technologie (support) : la nature de la technologie a un impact significatif sur les relations entre acteurs au sein d'une filière (Stieglitz et Heine, 2007). Lorsqu'une entreprise détient un support technique spécifique nécessaire à l'exécution de l'activité d'autres acteurs, elle dispose généralement d'un pouvoir de négociation plus fort (Kumar et Van Dissel, 1996). Ainsi, le support technique a une influence sur le degré d'interdépendance entre les parties (Dibiaggio, 1999). En effet, les acteurs situés en aval seront dans l'obligation d'exploiter le standard technologique développé en amont pour créer et diffuser leur produit.

Position au sein de la filière : dans une filière, il est possible de distinguer deux types d'acteurs : les acteurs situés en amont, qui sont généralement à l'origine de la création de la valeur et ceux situés en aval, qui disposent des capacités et des ressources pour diffuser et 


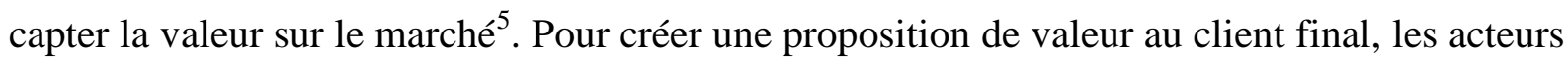
de l'amont sont donc contraints de collaborer avec les acteurs de l'aval. Ces derniers disposent alors d'un pouvoir de négociation plus important puisqu'ils permettent l'accès au marché (Fonrouge, 2007). Le pouvoir de négociation est également influencé par le degré de concentration des acteurs. Plus le marché est oligopolistique plus le risque de dépendance est élevé (Nooteboom et al., 2000).

Ces différentes sources (type et niveau de ressources et compétences, taille de l'entreprise, nature de la technologie et position dans la filière) déterminent le degré de dépendance des acteurs au sein de la filière et les conditions de l'échange (Cook, 1977). Le pouvoir de négociation des parties influence l'issue de la négociation (Schelling, 1956) et les règles de partage de la valeur. C'est pourquoi les entreprises fortement dépendantes tentent de mettre en œuvre des stratégies pour limiter leur dépendance et capter davantage de valeur. Toutefois, nous pensons que le choix stratégique (coopération ou intégration) peut être subi et dépend fortement des sources de dépendance.

\subsection{STRATEGIES POUR DIMINUER SON DEGRE DE DEPENDANCE}

Rares sont les entreprises capables de maîtriser l'intégralité des activités au sein de leur filière. Cette situation implique des phénomènes de dépendance entre acteurs. Pour minimiser cette dépendance, les entreprises peuvent recourir à l'intégration de ressources et compétences clés et/ou aux coopérations (Wang et Zajac, 2007) ${ }^{6}$.

Coopération $^{7}$ : la coopération unit des entreprises indépendantes dans un projet commun, établi dans une perspective de durée (Ingham, 1994). Les entreprises coopèrent principalement pour accélérer l'expansion tout en diminuant les coûts et les risques, réduire la concurrence et accéder aux compétences des partenaires (Hagedoorn, 1993). Il s'agit par exemple de réunir des moyens financiers, de partager les risques liés au développement de nouveaux produits ou de combiner des expertises dispersées et de coordonner des stratégies de développement pour des produits de plus en plus interdépendants (Segrestin, 2006). La coopération peut être réalisée avec une entreprise concurrente ou avec une entreprise située en amont et/ou en aval de la filière (Wang et Zajac, 2007). La signature d'un contrat de

\footnotetext{
${ }^{5}$ Cette distinction est également présente dans les travaux de Chesbrough (2003a) qui différencie les acteurs à l'origine de la création de valeur et ceux qui en assurent la captation.

${ }^{6}$ De multiples stratégies peuvent être instaurer par les entreprises : diversification, évitement, soumission, etc. (Pfeffer et Salancik, 1978). Toutefois, dans cet article nous nous focalisons sur le choix de leur mise en œuvre qui passe principalement soit par la coopération soit par l'intégration (Wang et Zajac, 2007).

${ }^{7}$ Dans cette recherche, nous considérons la coopération comme une forme organisationnelle à part entière (Powell, 1990) et non comme état transitoire (Williamson, 1985).
} 
coopération avec une entreprise située en aval permet d'être plus proche du marché et d'avoir une meilleure connaissance des besoins et attentes des clients finaux (Mohr et Spekman, 1994). A contrario, une coopération avec une entreprise située en amont favorise la maîtrise de la technologie. Enfin, le recours à la coopération avec un concurrent permet, notamment aux entreprises de petite taille, de proposer une offre plus diversifiée et d'avoir un pouvoir de négociation plus important face aux acteurs situés en amont et en aval de la filière. L'instauration de relations de coopération apparaît donc comme un moyen privilégié pour diminuer le degré de dépendance d'une entreprise au sein de sa filière. Toutefois, cette stratégie n'est pas exempte de contraintes (Koenig, 1990), de difficultés et engendre des coûts de coordination élevés. De plus, un niveau de confiance insuffisant entre les partenaires peut engendrer des comportements individualistes (Hamel et al., 1989). Afin de pallier ces difficultés, certaines entreprises peuvent être amenées à privilégier l'intégration pour diminuer leur degré de dépendance.

L'intégration de ressources et compétences en interne. Trois principales possibilités existent pour intégrer de nouvelles ressources et compétences : former le personnel de l'entreprise, recruter des personnes extérieures (exemple: démarcher un salarié d'une entreprise possédant les ressources et compétences recherchées) et acquérir une entreprise.

L'internalisation de nouvelles ressources et compétences permet d'éviter les risques d'opportunisme, de favoriser l'émergence d'un langage commun au sein de l'entreprise et d'éviter les divergences d'objectifs (Williamson, 1985). En effet, dans le cadre de relations interorganisationnelles, les acteurs sont constamment exposés au risque potentiel de se voir retirer une partie de la valeur créée. Cette situation, qualifiée de hold-up (Castaneda, 2006 ; Klein et al., 1978), découle d'une protection insuffisante des actifs. Dans une filière, les investissements spécifiques peuvent être réalisés par un ou plusieurs acteurs de cette filière (Dyer, 1997). En ayant recours à l'internalisation des activités à forte valeur ajoutée, les acteurs minimisent ce risque d'opportunisme. L'intégration verticale apparaît aussi comme un moyen d'accroître son pouvoir de négociation (Porter, 1985), de réduire les coûts de transaction (Williamson, 1985) et de multiplier les gisements de revenus (Warnier et al., 2006). L'entreprise peut aussi faire le choix de recourir aux fusions/acquisitions pour intégrer de nouvelles ressources. Toutefois, l'intégration d'une entreprise ayant des modes de fonctionnement différents (leadership, culture, structure, technologie, système de gestion et de communication) peut engendrer des conflits (Busija, O’Neill et Zeithaml, 1997). Par ailleurs, quelque soit la solution choisie pour intégrer de nouvelles ressources, l'intégration est souvent plus coûteuse que la coopération (Wang et Zajac, 2007). Se concentrer sur son cœur de métier 
plutôt que d'acquérir des ressources et compétences connexes peut permettre de réaliser des économies d'échelles. De plus, un acteur indépendant sera probablement plus motivé qu'un acteur intégré et assuré de sa survie ( Nooteboom et al., 2000).

\begin{tabular}{|c|c|c|}
\cline { 2 - 3 } \multicolumn{1}{c|}{} & Principaux avantages & Principaux inconvénients \\
\hline $\begin{array}{c}\text { Réalisation } \\
\text { d'une } \\
\text { coopération }\end{array}$ & $\begin{array}{c}\text { - Economies d'échelles } \\
\text { - Incitation plus forte du partenaire } \\
\text { indépendant à collaborer (motivation) } \\
\text { - Peu coûteuse }\end{array}$ & $\begin{array}{c}\text { - Coûts liés à la sortie anticipée d'un } \\
\text { partenaire pour cause de conflit ; } \\
\text { - Comportements opportunistes (ex : un } \\
\text { partenaire détourne plus de valeur qu'il } \\
\text { n'en produit) }\end{array}$ \\
\hline $\begin{array}{c}\text { Intégration } \\
\text { de }\end{array}$ & $\begin{array}{c}\text { - Réduit l'incertitude : risque } \\
\text { d'opportunisme }\end{array}$ & $\begin{array}{c}\text { - Risque de dispersion et de dilution du } \\
\text { couur de métier }\end{array}$ \\
$\begin{array}{c}\text { ressources et } \\
\text { compétences } \\
\text { en interne }\end{array}$ & $\begin{array}{c}\text { - Faibles coûts de coordination } \\
\text { - Accrô̂t son pouvoir de négociation } \\
\text { - Multiplier les sources de revenus }\end{array}$ & $\begin{array}{c}\text { - Souvent coûteuse à mettre en ouvre } \\
\text { - Risque de conflits en cas de } \\
\text { fusion/acquisition }\end{array}$ \\
\hline
\end{tabular}

Tableau 1 : Synthèses des principaux avantages et inconvénients de ces deux stratégies

Le tableau 1 fait apparaître qu'aucune de ces deux stratégies pour diminuer son degré de dépendance n'est optimale et dépend du contexte dans lequel l'entreprise évolue. Or, même si dans certaine situation, la coopération peut être perçue comme le meilleur moyen pour atteindre les objectifs, l'entreprise n'a pas toujours la possibilité de le faire. Le choix stratégique semble pouvoir être subi et nous tentons ici de comprendre l'influence des sources de dépendance sur ce choix. La figure 1 ci-dessous synthétise notre cadrage conceptuel qui nous servira de base pour notre étude empirique, réalisée dans le secteur des jeux vidéo.

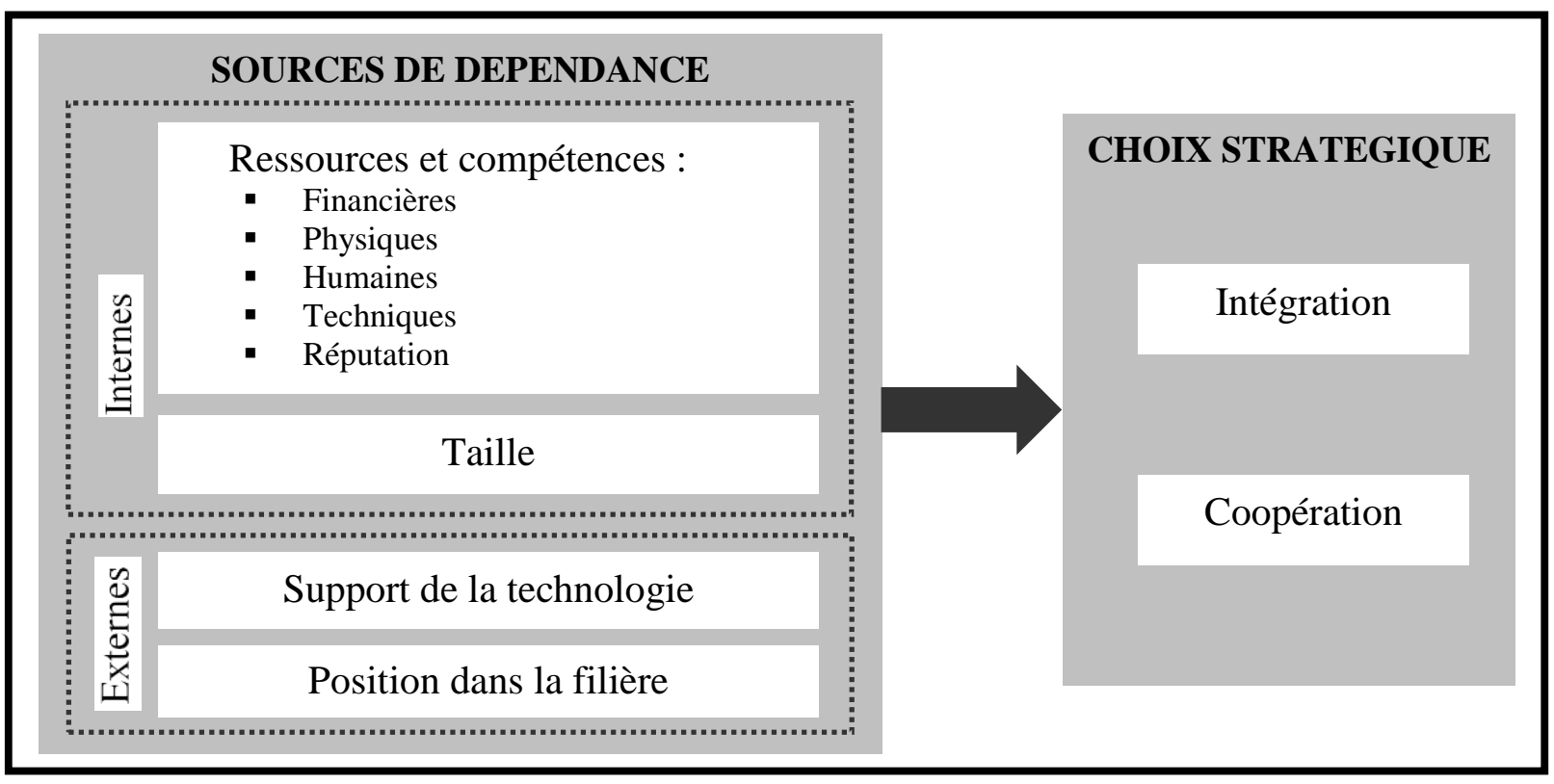

Figure 1 : Cadre conceptuel des sources de dépendance et des stratégies mises en œuvre 


\section{INDUSTRIE DU JEU VIDEO}

Cette recherche se focalise sur l'industrie du jeu vidéo qui présente la caractéristique d'être composée d'entreprises hétérogènes (taille, cœur de métier, technologie, etc.) particulièrement sujettes aux logiques de dépendance. Ce choix et les spécificités de cette industrie, notamment en matière de dépendance, sont détaillées (section 2.1). Nous présentons ensuite les cinq études de cas (section 2.2.) ainsi que les données recueillies et leur traitement.

\subsection{CARACTERISTIQUES ET SPECIFICITES DE L'INDUSTRIE DU JEU VIDEO}

Datant des années 80, l'industrie du jeu vidéo ${ }^{8}$ est aujourd'hui un acteur principal des industries créatives (Kinder, 1991 ; Hermann, 1999). Assimilé à un produit culturel, le jeu vidéo est un mélange complexe de technologie, d'art et d'interactivité (Cohendet et Simon, 2007 ; Rouet, 2009). Il implique un régime d'innovation soutenu (forte créativité et originalité) soumis à des contraintes temporelles et concurrentielles élevées (Storz, 2008). Ces spécificités ont des conséquences importantes sur l'offre comme sur la demande. D'une part, elles sont génératrices de risques économiques mais également psychologiques et sociaux pour les entreprises. D'autre part, ces spécificités induisent une incertitude dans le choix du consommateur (Busson et Evrard, 1987). Elles impliquent également de fortes contraintes organisationnelles au niveau du processus de production et de l'équilibre entre les aspirations « artistiques » et les nécessités « gestionnaires » (Gil et Spiller, 2007).

Le processus de création d'un jeu vidéo est composé de plusieurs phases (Parmentier et Mangematin, 2009) en amont (la conception, la pré-production, la production et la postproduction) et en aval (la commercialisation, le marketing et la distribution). Ces activités se répartissent entre quatre grands types d'acteurs (Le Diberder, 2002): les fabricants de consoles, les studios, les éditeurs et les distributeurs. Les studios, de par leur position au sein de la filière, se retrouvent en situation de dépendance tant par rapport aux acteurs de l'amont que de l'aval. En outre, ils sont présents sur les trois segments du marché: jeux sur consoles (consoles de salons et portable), sur PC et sur Internet et téléphones mobiles ${ }^{9}$. Ces deux raisons nous conduisent à nous focaliser sur ces acteurs pour étudier de quelle manière ils diminuent leur degré de dépendance.

\footnotetext{
${ }^{8}$ Selon une estimation de l'AFJV (Association Française pour le Jeu Vidéo), le marché mondial du logiciel de jeux vidéo, toutes plates-formes confondues, s'élevait à plus de 35 milliards d'euros en 2008. En France, le marché a franchi la barre des trois milliards d'euros en 2008 et compte aujourd'hui près de 330 entreprises dans ce secteur.

${ }^{9}$ Selon l'AFJV (2009), les jeux sur téléphones mobiles sont de plus en plus téléchargés depuis Internet. C'est pourquoi les opérateurs et constructeurs de téléphones proposent de plus en plus d'application de téléchargement. Dans cette perspective, nous préférons regrouper ici ces deux segments du marché.
} 


\subsection{ETUDES DE CAS DE CINQ STUDIOS}

Cette recherche vise à mieux comprendre les sources de dépendance influençant les « choix » stratégiques adoptés. L'étude exploratoire permet d'avoir une vision complète des causes d'un phénomène et, éventuellement, de leurs interactions (Miles et Huberman, 2003). Enfin, elle permet de répondre à un objectif de contextualisation de la recherche. Cette recherche, de nature prescriptive, nécessite différents cas comparables. Les studios retenus doivent être suffisamment parents (les cinq studios possèdent en interne un savoir-faire dans le développement de jeux vidéo sur consoles ou sur un autre type de plateforme) pour autoriser une comparaison pertinente tout en étant suffisamment distincts. Les différences (la taille, la détention des DPI, la stratégie, la réputation, etc.) donnent la possibilité de contrôler les influences idiosyncrasiques de chacun.

Afin d'analyser les sources de dépendance influençant le choix stratégique des entreprises, nous avons étudié, entre septembre 2008 et octobre 2009, cinq studios de tailles différentes ${ }^{10}$. Des accords de confidentialité ayant été signés, nous avons utilisé des pseudonymes pour les désigner : la société «Flashgame », «Evolugame », «Créajeux », «Jémulex» et la société «MOP (Multi Online Players)». Notre recherche qualitative exploite des données primaires issues de 18 entretiens semi-directifs (d'une durée comprise entre $1 \mathrm{~h}$ et $1 \mathrm{~h} 30)$ et des données secondaires recueillies sur Internet et dans des journaux professionnels (cf. Tableau 2) ${ }^{11}$.

\begin{tabular}{|c|c|c|c|c|c|c|}
\hline \multicolumn{2}{|c|}{ Caractéristiques } & Flashgame & Evolugame & Créajeux & Jémulex & MOP \\
\hline & CA en 2008 & $1,05 \mathrm{M} €$ & $2 \mathrm{M} €$ & $17 \mathrm{M} €$ & $1,6 \mathrm{M} €$ & $1,5 \mathrm{M} €$ \\
\cline { 2 - 7 } & $\begin{array}{c}\text { Taille } \\
\text { (employés) }\end{array}$ & 17 & 40 & 500 & 12 & 24 \\
\cline { 2 - 7 } & $\begin{array}{c}\text { Ressources et } \\
\text { compétences } \\
\text { possédées en } \\
\text { interne }\end{array}$ & Productive & $\begin{array}{c}\text { Financière } \\
\text { (faible) et } \\
\text { productive }\end{array}$ & $\begin{array}{c}\text { Financière } \\
\text { (forte) et } \\
\text { productive }\end{array}$ & $\begin{array}{c}\text { Financière } \\
\text { (moyenne), } \\
\text { technologique } \\
\text { et productive }\end{array}$ & $\begin{array}{c}\text { Financière } \\
\text { (forte), } \\
\text { technologique } \\
\text { et productive }\end{array}$ \\
\cline { 2 - 7 } & $\begin{array}{c}\text { Détention des } \\
\text { droits de PI }\end{array}$ & Non & Non & Oui & Oui & Oui \\
\cline { 2 - 7 } & Valeur captée & Très faible & Faible & Forte & Moyenne & Moyenne \\
\cline { 2 - 7 } & $\begin{array}{c}\text { Relations au } \\
\text { sein de la } \\
\text { filière }\end{array}$ & $\begin{array}{c}\text { Fabricants et } \\
\text { éditeurs }\end{array}$ & $\begin{array}{c}\text { Fabricants et } \\
\text { éditeurs }\end{array}$ & $\begin{array}{c}\text { Fabricants, } \\
\text { éditeurs et } \\
\text { distributeur }\end{array}$ & Distributeurs & Fabricants \\
\cline { 2 - 7 } & $\begin{array}{c}\text { Nature de la } \\
\text { technologie }\end{array}$ & $\begin{array}{c}\text { Consoles } \\
\text { portables }\end{array}$ & $\begin{array}{c}\text { Consoles de } \\
\text { salons }\end{array}$ & $\begin{array}{c}\text { Consoles tous } \\
\text { types et PC }\end{array}$ & PC & $\begin{array}{c}\text { Internet sur } \\
\text { consoles }\end{array}$ \\
\hline
\end{tabular}

\footnotetext{
${ }^{10}$ Une description détaillée des cas est disponible sur demande.

${ }^{11}$ Pour des motifs de taille de la communication, nous n'avons pas inséré d'annexe méthodologique détaillant la méthode de recueil, de codage, de traitement et d'analyse des données. Cette annexe est disponible sur demande.

${ }^{12}$ Pourcentage des retours économiques perçus (royalties).
} 


\begin{tabular}{|c|c|c|c|c|c|c|}
\hline \multirow{4}{*}{ 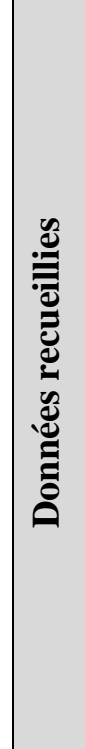 } & $\begin{array}{c}\text { Nombre } \\
\text { d'entretiens }\end{array}$ & 3 & 3 & 5 & 3 & 4 \\
\hline & $\begin{array}{l}\text { Personnes } \\
\text { interviewées }\end{array}$ & $\begin{array}{l}\text { - Directeur } \\
\text { commercial (1) } \\
\text { - Responsables } \\
\text { d'équipe (2) } \\
\end{array}$ & $\begin{array}{l}\text { - Directeur } \\
\text { technique (1) } \\
\text { - Responsables } \\
\text { artistique (2) }\end{array}$ & \begin{tabular}{|l} 
- Responsable \\
PI (2) \\
- Responsables \\
R\&D (3) \\
\end{tabular} & \begin{tabular}{|l} 
- Directeur du \\
studio (1) \\
- Responsables \\
technique (2) \\
\end{tabular} & $\begin{array}{l}\text { - Directeur de } \\
\text { la création (2) } \\
\text { - Responsables } \\
\text { projet (2) }\end{array}$ \\
\hline & $\begin{array}{c}\text { Données } \\
\text { internes }\end{array}$ & $\begin{array}{c}\text { Dossiers de } \\
\text { prototypage } \\
(2)+\text { cahier } \\
\text { des charges } \\
\text { projets (3) }\end{array}$ & $\begin{array}{c}\text { Documentation } \\
\text { projet }(5)+ \\
\text { courriers } \\
\text { éditeurs }(10)+ \\
\text { échanges de } \\
\text { courriels (4) } \\
\end{array}$ & $\begin{array}{c}\text { Contrats } \\
\text { éditeurs }(8)+ \\
\text { dossiers } \\
\text { projets }(4)+ \\
\text { échanges de } \\
\text { courriels }(11) \\
\end{array}$ & $\begin{array}{c}\text { Rapport } \\
\text { statistiques } \\
\text { d'abonnées aux } \\
\text { jeux (16) + } \\
\text { échanges de } \\
\text { courriels (3) } \\
\end{array}$ & $\begin{array}{l}\text { Rapport de } \\
\text { réunion projet } \\
\qquad(5)+ \\
\text { échanges de } \\
\text { Courriels (8) }\end{array}$ \\
\hline & $\begin{array}{l}\text { Données } \\
\text { externes }\end{array}$ & $\begin{array}{l}\text { Site Internet + } \\
\text { articles de } \\
\text { presses } \\
\text { numérique (3) }\end{array}$ & $\begin{array}{l}\text { Site Internet + } \\
\text { articles de } \\
\text { presses } \\
\text { numérique (4) }\end{array}$ & $\begin{array}{c}\text { Sites Internet } \\
(2)+\text { articles } \\
\text { de presse } \\
\text { numériques } \\
(14)\end{array}$ & $\begin{array}{l}\text { Site Internet + } \\
\text { articles de } \\
\text { presse } \\
\text { numériques (7) }\end{array}$ & $\begin{array}{l}\text { Sites Internet }+ \\
\text { articles de } \\
\text { presse } \\
\text { numériques (4) }\end{array}$ \\
\hline
\end{tabular}

Tableau 2 : Présentation des cinq studios étudiés et données recueillies

Nous avons réalisé un codage thématique des données issues des cinq studios. L'objectif est de comprendre les sources de dépendance et leur influence sur les stratégies mises en œuvre. Un extrait du codage des données est illustré dans l'annexe A. L'analyse des données ainsi traitées est présentée dans la section suivante.

\section{DEPENDANCE DES STUDIOS DE JEUX VIDEO}

Nous présentons les résultats issus des cinq études de cas en trois temps ${ }^{13}$. Tout d'abord, nous mettons en exergue le rôle clé des ressources et compétences par rapport aux autres sources de dépendance des studios (3.1.). Nous montrons ensuite que les sources de dépendance influencent le choix stratégique (3.2.). Enfin, nous discutons du lien entre source de la dépendance et stratégie mise en œuvre (3.3.).

Les contraintes issus des caractéristiques culturelles de l'environnement industriel :

\subsection{PRINCIPALES SOURCES DE DEPENDANCE DANS LE JEU VIDEO ${ }^{14}$}

Majoritairement, de par leur position au sein de la filière, les studios sont soumis à un fort degré de dépendance. Les sources de cette dépendance sont multiples. Au regard des cinq cas étudiés, nous avons classé les différentes sources de dépendance en fonction de leur niveau d'importance. Parmi celles-ci, le manque de ressources financières apparaît comme la première source de dépendance. La dépendance financière est essentiellement constatée avec les acteurs situés en aval (éditeurs et distributeurs) et s'accompagne d'une dépendance

\footnotetext{
13 Afin de respecter les contraintes de taille de l'article, nous ne présentons ici que quelques verbatims pour illustrer les principaux résultats.

${ }^{14}$ Dans cette section, nous analysons les sources de dépendance des studios étudiés avant qu'une stratégie soit mise en œuvre pour minimiser cette dépendance.
} 
éditoriale $^{15}$. Ce manque de ressources financières est intimement associé à la cession des DPI, autre source importante de dépendance. D'autres sources comme la nature du support, s'observent principalement avec les acteurs situés en amont (fabricants et fournisseurs de middleware). Enfin, la taille de l'entreprise apparaît comme la dernière source de dépendance.

Le manque de ressources financières : pour obtenir des ressources financières nécessaires au développement de jeux vidéo, trois des studios étudiés (Flashgame, Evolugame et Créajeux) ont recours aux éditeurs. Ce financement permet à la fois d'assurer la survie des studios et d'externaliser le risque lié à leur production ${ }^{16}$. Toutefois, le financement extérieur présente des inconvénients. Lorsqu'un éditeur finance l'ensemble du développement d'un jeu créé par un studio, ce dernier est contraint de céder l'intégralité des DPI au financeur. C'est le cas des studios Flashgame, Evolugame qui agissent en simple prestataire - alors qu'ils sont à l'origine de l'idée et du concept. Les éditeurs leur imposent également des contraintes éditoriales fortes qui diminuent leur liberté créative :

«Avec un éditeur, on peut pas vraiment faire ce qu'on veut, il faut respecter ses directives artistiques si on veut garder le financement» (Directeur commercial Flashgame).

Pour Créajeux, le financement des jeux par des acteurs extérieurs est partiel car le studio possède suffisamment de ressources financières pour participer au financement. Son degré de dépendance face aux éditeurs est donc moins élevé que celui de Flashgame et Evolugame. Pour les jeux sur PC, Créajeux s'adresse directement à un distributeur car le studio adapte systématiquement ses jeux consoles en jeux $\mathrm{PC}^{17}$. Créajeux n'est alors plus dépendant d'un éditeur mais d'un distributeur. Dans le cas de Jémulex et MOP, la dépendance en aval avec les éditeurs n'a pas été observée car les studios sont positionnés sur le marché des jeux PC et Internet sur console. Les jeux sur PC nécessitent moins d'investissements que ceux sur consoles et permettent l'utilisation de technologies moins coûteuses. Les jeux Internet sur consoles sont également moins coûteux car ils ne nécessitent pas de communication «classique » (publicité TV, etc.) et la diffusion est dématérialisée. En ce qui concerne la dépendance aval avec les distributeurs, seul Jémulex est dépendant car il ne dispose pas d'assez de ressources financières pour distribuer ses jeux PC aux grossistes et détaillants. Pour MOP, la diffusion des jeux directement sur Internet lui évite d'avoir recours à un distributeur. La dépendance aval est donc double : dépendance financière et éditoriale qui

\footnotetext{
${ }^{15}$ Dans le jeu vidéo, le terme «éditorial » désigne tous les éléments relatifs au contenu et à la qualité du jeu. C'est à dire, les éléments scénaristiques (histoire, scénario, etc.), les éléments graphiques et artistiques (personnage, décors, etc.) et les éléments de programmation du jeu (les différents niveaux, l'interactivité, etc.).

${ }^{16}$ Dans ce secteur, le risque d'échec est relativement élevé et le coût de conception et développement d'une production peut être élevé (plusieurs dizaine de millions d'euros pour un jeu sur console).

${ }^{17}$ L'adaptation est purement technologique puisque le jeu est déjà créé. L'investissement est donc minime.
} 
limite la liberté créative. De par les spécificités du secteur culturel (cf. 2.1.), la liberté créative est le fer de lance des studios puisqu'il s'agit de leur cœur de métier.

La cession des DPI : pour conserver les DPI, les studios doivent participer au financement (même partiellement). Cela permet d'augmenter leur pouvoir de négociation face aux éditeurs. Par ailleurs, la détention des DPI augmente la valeur intrinsèque des studios et contribue à construire leur réputation au sein de l'industrie. Pour Flashgame et Evolugame, la perte des DPI est systématique car ils ne sont pas en mesure de participer au financement des jeux. Ils sont donc fortement dépendants des éditeurs. A l'inverse, Créajeux ayant la possibilité de financer partiellement le développement de ses jeux sur consoles (la conception et la pré-production), a donc la possibilité de conserver ses DPI. Il reste toutefois partiellement dépendant des éditeurs qui continuent à financer la production et la postproduction. De plus, les contraintes éditoriales ne sont plus imposées mais négociées avec le studio, ce qui augmente cette liberté éditoriale :

«Depuis le début, on essaie de privilégier le développement de nos propres propriétés intellectuelles, cela demande plus d'investissement et de risque mais on récupère plus d'argent et on conserve nos droits; et ça c'est très important pour nous » (Responsable PI - Créajeux)

C'est la détention des DPI de ses jeux sur consoles qui permet à Créajeux de les adapter en jeux PC. Jémulex et MOP conservent systématiquement leurs DPI puisqu'ils financent l'intégralité du développement des jeux. Les éditeurs ne sont donc plus des intermédiaires.

La nature de la technologie (le support) : pour créer et développer les jeux sur consoles, les studios doivent utiliser les supports et standards technologiques ${ }^{18}$ imposées par les fabricants de consoles. C'est le cas des studios Flashgame, Evolugame, Créajeux et MOP. Ils sont présents sur console, malgré le risque de dépendance, car il s'agit du segment le plus rentable de l'industrie (AFJV, 2009). Ils acceptent ainsi ce fort degré de dépendance afin d'assurer la pérennité et la croissance de leur entreprise :

«Pour développer des jeux sur consoles, on est obligé de travailler avec les fabricants, on doit utiliser la technologie qu'ils nous fournissent pour développer les jeux mais aussi prouver qu'on est capable de s'en servir pour développer de bons jeux; sinon, on est pas considéré comme développeur agréé et personne ne va vouloir travailler avec nous. » (Directeur technique - Evolugame).

En revanche, les performances artistiques de leurs jeux sont limitées par les capacités techniques des consoles. Leur liberté créative est donc diminuée car le personnel créatif doit travailler dans un environnement technologique contraint (par les consoles). Jemulex n'utilise pas la technologie des fabricants puisqu'il ne développe pas de jeux sur consoles mais sur PC.

${ }^{18}$ Il s'agit de la plateforme de diffusion (la console) ainsi que le moteur 3D permettant de programmer le jeu dans le respect des capacités techniques de la plateforme. Comme ces technologies sont propriétaires (brevets, etc.), les studios sont obligés de subir la dépendance des fabricants pour y accéder. 
La technologie utilisée est moins coûteuse et plus «accessible ${ }^{19}$ ». Elle permet ainsi de se libérer des standards imposés par les fabricants de consoles :

«Nous, on ne s'embête pas, on a décidé dès le début de programmer sur PC, ce qui nous permet d'être libre et même de créer ensuite notre propre technologie » (Directeur du studio - Jémulex)

C'est également le cas de Créajeux qui réalise des PC. Jémulex n'est donc pas en situation de dépendance face aux acteurs de l'amont et Créajeux l'est uniquement sur le segment des jeux consoles. Les studios peuvent ainsi augmenter leur liberté créative devant les multiples possibilités technologiques proposées par les autres supports (PC, Internet, etc.) et évitent la dépendance de l'amont.

Position au sein de la filière : un studio peut modifier sa position dans la filière s'il parvient à intégrer des ressources. C'est le cas de Jémulex et MOP mais également de Créajeux pour ses jeux PC. Jémulex a intégré des ressources humaines, techniques et financières lui permettant de réaliser des jeux sur PC et de financer l'intégralité de leur développement sans avoir besoin de recourir aux fabricants ni aux éditeurs. Jémulex reste néanmoins partiellement dépendant des distributeurs. Pour MOP, le studio a intégré des ressources humaines et financières lui permettant d'assurer l'ensemble du développement et de la distribution de ses jeux sur Internet sans l'aide financière d'éditeurs et sans distributeur :

«Malgré notre taille, nous sommes assez contents de pouvoir financer complètement nos jeux car cela démontre notre autonomie » (Directeur de la création - MOP)

Malgré cela, MOP reste partiellement dépendant des fabricants de consoles. Créajeux évite la dépendance des fabricants et des éditeurs sur le segment des jeux PC puisqu'il finance seul l'adaptation de ses jeux consoles en jeux PC. La seule dépendance à laquelle il est soumis est celle des distributeurs. Aucun des deux autres cas (Flashgame et Evolugame) n'a tenté d'intégrer des compétences amont ou aval pour modifier sa position dans la filière. Ils restent donc dépend des acteurs situés en amont et en aval de leur position.

Parmi les ressources et compétences des studios, l'absence de détention des droits de PI et de réputation apparaissent comme des sources clés de la dépendance.

L’absence de réputation : elle n'a d'influence que sur la dépendance éditoriale :

«Notre réputation dans le milieu compte beaucoup pour nous, c'est notre carte de visite, on décroche plus de projets ou on nous fait plus confiance; mais ce n'est pas pour autant qu'on est totalement libre de faire ce qu'on veut » (Directeur technique - Evolugame)

Dans le cas d'Evolugame et Créajeux, leur réputation permet d'augmenter leur crédibilité et leur «image » face aux éditeurs. La négociation est alors facilitée car la réputation du studio

\footnotetext{
${ }^{19}$ On parle ici de technologies qu'il est possible d'acheter ou des technologies libres de droit comme la technologie disponible sur Internet et permettant de faire des jeux à un coût très bas sur PC ou sur Internet.
} 
apporte aux éditeurs une garantie sur le succès potentiel du jeu vidéo (surtout pour Créajeux qui dispose d'une forte réputation). En contrepartie, l'éditeur accorde plus de confiance au studio et diminue ainsi ses contraintes éditoriales. Pour Flashgame, sa réputation est encore quasi inexistante du fait de sa création récente (2004). Pour Jémulex et MOP, il n'y a pas d'éditeurs mais leur réputation est établit, grâce en partie, au succès de leurs produits.

La taille du studio : dans les cinq studios étudiés (de taille différente), celle-ci n'apparaît pas comme une source importante de dépendance. Jémulex par exemple est un studio de petite taille qui est peu dépendant des autres acteurs :

«Notre petite taille n'est pas un handicap, on tient à rester petit pour être flexible et d'ailleurs on n'a de compte à rendre à personne sauf aux distributeurs » (Directeur $\mathrm{du}$ studio - Jémulex)

A contrario, Créajeux est fréquemment en situation de dépendance malgré sa taille importante car il interagit avec l'ensemble des acteurs de la filière (fabricants, les éditeurs et les distributeurs) lorsqu'il développe des jeux sur consoles. Ces sources de dépendance sont synthétisées dans le Tableau 3 ci-après :

\begin{tabular}{|c|c|c|c|}
\hline \multirow{5}{*}{ 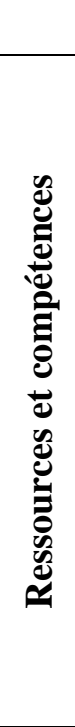 } & & \multirow{2}{*}{\begin{tabular}{|l|}
\multicolumn{1}{|c|}{ N'est pas source de dépendance si } \\
Le studio a suffisamment de ressources \\
financières pour assurer l'ensemble du \\
processus de production et de diffusion. \\
(cf. MOP)
\end{tabular}} & Source de dépendance si \\
\hline & Financières & & $\begin{array}{l}\text { Pas assez de ressources financières pour } \\
\text { exercer l'ensemble de son activité. } \\
\text { (cf. Flashgame, Evolugame, Créajeux, } \\
\text { Jémulex) }\end{array}$ \\
\hline & $\begin{array}{l}\text { Techniques } \\
\text { (DPI) }\end{array}$ & $\begin{array}{l}\text { Le studio est seul pour financer et } \\
\text { développer de son jeu. Il conserve donc } \\
\text { l'intégralité des droits de PI. } \\
\text { (cf. MOP et Jémulex) }\end{array}$ & $\begin{array}{l}\text { Le studio a besoin de céder une partie ou } \\
\text { l'intégralité des droits de PI à des acteurs } \\
\text { extérieurs pour financer son jeu. } \\
\quad \text { (cf. Flashgame, Evolugame, Créajeux) }\end{array}$ \\
\hline & Réputation & \multicolumn{2}{|c|}{$\begin{array}{l}\text { La réputation a un impact sur la crédibilité des studios face aux éditeurs. Elle permet de } \\
\text { diminuer la dépendance éditoriale mais ne la supprime pas (cf. Créajeux et Evolugame). } \\
\text { En l'absence de réputation, le studio n'a pas de pouvoir de négociation face aux éditeurs } \\
\text { (cf. Flashgame). }\end{array}$} \\
\hline & & \multicolumn{2}{|c|}{$\begin{array}{l}\text { La taille de l'entreprise ne semble pas être une source de dépendance. Une entreprise de } \\
\text { petite taille peut être moins dépendante qu'une entreprise de grande taille. } \\
\text { (cf. Jémulex versus Créajeux) }\end{array}$} \\
\hline \multicolumn{2}{|c|}{$\begin{array}{l}\text { Nature de la } \\
\text { technologie } \\
\text { (support) }\end{array}$} & $\begin{array}{l}\text { Le support et les standards techniques } \\
\text { utilisés ne sont pas ceux imposés par les } \\
\text { fabricants de consoles. } \\
\text { (cf. Jémulex et Créajeux) }\end{array}$ & $\begin{array}{l}\text { Le support et les standards techniques utilisés } \\
\text { sont fournis par les fabricants de consoles. } \\
\text { (cf. Flashgame, Evolugame, Créajeux, MOP) }\end{array}$ \\
\hline \multicolumn{2}{|c|}{$\begin{array}{l}\text { Position au sein } \\
\text { de la filière }\end{array}$} & $\begin{array}{l}\text { Le studio parvient à maîtriser l'ensemble } \\
\text { des activités de la filière ou à se } \\
\text { positionner sur un marché dans lequel les } \\
\text { principaux acteurs de la filière sont } \\
\text { absents comme Internet (mais pas le } \\
\text { marché Internet sur consoles). }\end{array}$ & $\begin{array}{l}\text { Le studio ne maitrise pas toutes les activités } \\
\text { de la filière. En fonction de sa position, il peut } \\
\text { être soumis à une dépendance en aval (cf. } \\
\text { Jémulex) ou en amont (cf. MOP) ou en amont } \\
\text { et en aval. } \\
\text { (cf. Flashgame, Evolugame, Créajeux). }\end{array}$ \\
\hline
\end{tabular}

Tableau 3 : Principales sources de dépendance 
Ces cinq studios sont soumis à différentes sources de dépendance. Dans la section suivante, nous tentons de comprendre l'influence de ces multiples sources de dépendance sur le choix stratégique.

\subsection{SOURCES DE DEPENDANCE INFLUENÇANT LE CHOIX STRATEGIQUE}

Pour augmenter leur pouvoir de négociation et leur liberté éditoriale, les studios ont recours à deux principales stratégies : la coopération ou l'intégration de ressources et compétences.

Les sources de dépendance influençant le recours à la coopération : la coopération avec un acteur situé en amont ou en aval de la filière n'a jamais été observée ${ }^{20}$. En amont, seuls trois fabricants de consoles se partagent le marché (Nintendo, Sony et Microsoft) et le coût de la technologie (support) créer des barrières à l'entrée difficilement surmontables. De plus, une masse critique est nécessaire pour développer un standard technologique. En aval, même s'il apparait bénéfique pour un studio de coopérer avec des éditeurs, cette situation n'a pas non plus été observée. En effet, avoir une relation exclusive avec un seul éditeur les rendrait encore plus fortement dépendants de ce dernier. Ainsi, dans les deux cas où la coopération a été observée (Flashgame et Evolugame), il s'agit d'une coopération avec des concurrents.

Flashgame est un petit studio soumit à plusieurs sources de dépendance. Tout d'abord, son manque de ressources financières l'oblige à recourir aux éditeurs. Ce recours contraint systématiquement Flashgame à céder ses DPI. Enfin, son absence de réputation ne favorise pas les négociations avec les éditeurs. Face à ces sources de dépendance, le studio se lance en 2006 dans un projet de co-création de jeux avec un studio de taille moyenne qui désire se diversifier sur les consoles portables. Pour Flashgame, l'objectif premier est de diminuer sa dépendance face aux éditeurs. Toutefois, l'absence de réputation de Flashgame l'oblige, dans un premier temps, à opter pour le recours à la coopération avec un studio plus « visible » :

«e choix du partenaire était très important pour nous. Nous, on lui apportait la compétence en termes de console portable et lui devait nous apporter plus, on voulait se faire connaître à travers lui » (Directeur commercial - Flashgame)

Le financement combiné des deux partenaires (30\% Flashgame et 70\% studio partenaire) leur permet de conserver les DPI car l'éditeur n'a pas besoin de financer la totalité du jeu. Toutefois, les retours économiques octroyés à Flashgame sont proportionnels à son investissement (30\% de la rémunération brut de l'éditeur). De plus, le potentiel créatif de Flashgame doit prendre en compte les directives artistiques de son partenaire car c'est lui qui porte le projet. Malgré ces contraintes, Flashgame accepte ces conditions et une répartition non égalitaire car elles lui permettent de se construire une réputation.

${ }^{20}$ Ils se limitent à des relations de filières. 
Dans le cas d'Evolugame, l'absence de réputation n'est pas une source de dépendance, seul le manque de ressources financières et la cession des DPI le sont. A la différence de Flashgame, Evolugame est un studio plus âgé qui s'est forgé une réputation au fil des années. Il peut donc négocier plus facilement avec les éditeurs mais reste toutefois dans une position forte de dépendance (notamment, à cause du besoin en financement). Pour faire face à ces contraintes, le studio entreprend en 2005 une coopération avec un studio de taille similaire afin de concevoir un prototype de jeu. L'objectif est de conserver les DPI et de limiter l'investissement de l'éditeur afin de diminuer son degré de dépendance. Grâce à cette stratégie, l'éditeur n'a financé que $40 \%$ du projet, les contraintes éditoriales ont été négociées et la liberté éditoriale des deux studios a été augmentée :

«C'est sur que l'éditeur n'a pas été aussi virulent qu'avant, là on avait vraiment la possibilité de faire ce qui nous passait par la tête »(Responsable artistique - Evolugame)

\begin{tabular}{|c|c|c|c|c|c|}
\hline & & \multirow{2}{*}{$\begin{array}{l}\text { Type de } \\
\text { support }\end{array}$} & \multicolumn{3}{|c|}{ Source de dépendance à l'origine du choix stratégique } \\
\hline & & & $\begin{array}{c}\text { Manque de ressources } \\
\text { financières }\end{array}$ & Cession des DPI & $\begin{array}{l}\text { Absence de } \\
\text { réputation }\end{array}$ \\
\hline \multirow{3}{*}{ Flashgame } & Avant & Console & +++ & +++ & +++ \\
\hline & $\begin{array}{c}\begin{array}{c}\text { Stratégie } \\
\text { mise en } \\
\text { euvre }\end{array} \\
\end{array}$ & \multicolumn{4}{|c|}{$\begin{array}{l}\text { Coopération avec un studio de plus grosse taille pour co-créer un jeu et } \\
\text { conserver une partie des droits de PI }(30 \%) \text {. }\end{array}$} \\
\hline & Après & Console & ++ & ++ & ++ \\
\hline \multirow{3}{*}{ Evolugame } & Avant & Console & ++ & ++ & - \\
\hline & \begin{tabular}{|c|}
$\begin{array}{c}\text { Stratégie } \\
\text { mise en } \\
\text { ouuve }\end{array}$ \\
\end{tabular} & \multicolumn{4}{|c|}{$\begin{array}{l}\text { Coopération avec un studio de même taille pour co-créer un jeu et } \\
\text { conserver les droits de PI (répartition égalitaire). }\end{array}$} \\
\hline & Après & Console & + & + & - \\
\hline
\end{tabular}

Tableau 4 : Stratégies de coopération misent en œuvre en fonction des sources de dépendance

Le tableau 4 montre que le manque de ressources financières, la cession des DPI et l'absence de réputation sont les sources de dépendance à l'origine du choix stratégique de Flashgame. La stratégie de coopération mise en œuvre a eu pour conséquence de diminuer la dépendance envers l'éditeur puisqu'une partie des droits a été conservée grâce au financement combiné des deux studios. Pour Evolugame, l'absence de réputation n'a pas été une source de dépendance influençant le choix stratégique car le studio bénéficiait déjà d'une réputation dans la filière. La stratégie de coopération mise en œuvre a été influencée uniquement par le besoin de financement et la volonté de ne pas céder les DPI à l'éditeur. 
Les sources de dépendance influençant le recours à l'intégration de ressources : parmi les cinq cas étudiés, trois (Créajeux, Jémulex et MOP) présentent des stratégies d'intégration de ressources et compétences financières (grâce au succès de leurs produits) et/ou humaines et techniques (permettant le développement et la diffusion de jeux sur Internet). L'adoption d'un autre support technologique a permis aux studios de modifier leur position dans la filière. En revanche, aucune intégration de ressources n'a été observée sur le marché des jeux consoles ${ }^{21}$.

Après quelques années, Créajeux devient un studio de grande taille qui participe au financement de ses jeux afin de conserver les DPI. Le manque de ressources financières n'est donc pas la source principale de dépendance mais plutôt une source secondaire puisque le studio a néanmoins recours aux éditeurs pour financer l'ensemble du développement. La source principale de dépendance de Créajeux est la nature du support technologique qu'il utilise pour créer ses jeux. Le studio est en effet en étroite relation avec les fabricants de consoles, ce qui l'oblige à respecter scrupuleusement leurs standards et leurs supports technologiques. Cette situation fait naître pour Créajeux une dernière source de dépendance : la position défavorable au sein de la filière. Pour réagir à ces sources de dépendance, le studio a acquis des ressources humaines et techniques afin d'adapter ses jeux consoles en jeux Internet et téléphones mobiles. Le studio a embauché une dizaine de personnes expertes dans ces technologies (programmation Internet et téléphones mobiles). Cette stratégie permet de se soustraire aux supports et standards des fabricants :

«On voulait essayer de se diversifier au maximum pour prolonger les expériences liées à nos jeux. En plus, on n'était plus obligé d'utiliser la technologie des fabricants : donc c'était vraiment un avantage pour nous » (Responsable R\&D - Créajeux)

Le financement total du jeu est possible car les coûts de production sont faibles. Pour la diffusion, le studio a d'abord utilisé son site Internet pour le téléchargement. Ce mode de distribution s'est avéré peu efficace (manque de visibilité) et Créajeux s'est tourné vers des sites spécialisés. Le studio est en situation de dépendance face à ces distributeurs. Toutefois, cette dépendance est limitée car ils n'ont pas d'exclusivité et son facilement substituables.

Dans la situation initiale de MOP, la source principale de dépendance est la nature du support technologique puisque le studio crée et développe des jeux à destination des plateformes Internet des consoles de salon ${ }^{22}$. Le coût de ce type de jeux étant moins important que les jeux «classiques » sur console, le studio parvient à financer entièrement ses jeux (le

\footnotetext{
${ }^{21}$ Ceci s'explique par la nécessité pour un studio, d'avoir une capacité financière extrêmement importante pour intégrer le métier de fabricant, d'éditeur ou de distributeur. Ubisoft est à notre connaissance, la seule entreprise ayant réussi ce défi. Toutefois, elle n'est pas étudié dans ce papier.

${ }_{22}$ Depuis 2002, avec la sortie du service Xbox Live, les consoles de salon et consoles portables bénéficient d'un service en ligne (plateforme Internet) permettant de télécharger des jeux et de jouer en réseau.
} 
recours aux éditeurs n'est donc pas nécessaire). Toutefois, sa position défavorable dans la filière (par rapport aux fabricants) s'avère être une deuxième source de dépendance. Pour améliorer sa situation, MOP a intégré, en 2007, des ressources humaines et techniques lui permettant de créer des $\mathrm{MMORPG}^{23}$ sur Internet. Grâce à ces ressources, MOP a réutilisé ses DPI afin de proposer une version modifiée de ses jeux et d'augmenter sa créativité :

«On a créé quelque chose de vraiment original grâce à Internet, même si ça ressemble un petit peu aux jeux qu'on faisait avant, on a quand même une grosse partie technologique complètement différente qui est beaucoup mieux » (Responsable projet - MOP).

Les jeux sont diffusés sur des sites Internet développés par le studio et la rentabilité est assurée par un système d'abonnement mensuel. La réputation du studio a aussi contribué au succès de ses nouveaux jeux sur Internet.

A la différence des autres studios étudiés, Jémulex crée et développe des jeux sur PC. La nature du support n'est donc pas une source de dépendance pour lui puisque le support utilisé n'est pas propriétaire comme pour les consoles. Sa principale source de dépendance est son manque de ressources financières qui ne lui permet pas d'assurer la distribution de ses jeux. Sa position défavorable au sein de la filière (par rapport aux distributeurs) est également une source de dépendance. Toutefois, Jémulex décide en 2004 de rendre disponible l'un de ses jeux à succès sur Internet (par téléchargement) afin d'en faire un jeu massivement multijoueurs (MMORPG). Le studio a alors intégré des ressources techniques (formation de ses employés) afin de créer un site Internet permettant la diffusion de son jeu. Celui-ci est accessible gratuitement sur Internet, mais cette version gratuite reste limitée. Pour accéder à l'ensemble des fonctionnalités, le joueur doit acheter en ligne la version complète du jeu. Aujourd'hui, Jémulex utilise uniquement Internet pour diffuser son média :

«Notre jeu se télécharge très bien sur Internet et on est vraiment très content que les joueurs se soit familiarisés avec notre site. Au final, c'est bien mieux que la distribution traditionnelle » (Responsable technique - Jémulex).

\begin{tabular}{|c|c|c|c|c|c|}
\hline & \multirow{2}{*}{$\begin{array}{l}\text { Type de } \\
\text { support }\end{array}$} & \multicolumn{3}{|c|}{ Source de dépendance à l'origine du choix stratégique } \\
\hline & & & $\begin{array}{l}\text { Manque de ressources } \\
\text { financières }\end{array}$ & $\begin{array}{c}\text { Nature du } \\
\text { support }\end{array}$ & $\begin{array}{l}\text { Position dans la } \\
\text { filière }\end{array}$ \\
\hline \multirow{3}{*}{ Créajeux } & Avant & Console & ++ & +++ & ++ \\
\hline & $\begin{array}{c}\text { Stratégie } \\
\text { mise en } \\
\text { ouvre }\end{array}$ & \multicolumn{4}{|c|}{$\begin{array}{l}\text { Intégration de ressources techniques pour diversifier les supports de diffusion } \\
\text { de ses jeux (Internet et téléphonie mobile). }\end{array}$} \\
\hline & Après & $\begin{array}{l}\text { Internet et } \\
\text { téléphone } \\
\text { mobile }\end{array}$ & - & - & + \\
\hline
\end{tabular}

\footnotetext{
${ }^{23}$ MMORPG : Massively Multiplayer Online Role Playing Games (Jeux de rôle en ligne massivement multijoueurs).
} 


\begin{tabular}{|c|c|c|c|c|c|}
\hline \multirow{3}{*}{ MOP } & Avant & Console & - & +++ & + \\
\hline & $\begin{array}{c}\text { Stratégie } \\
\text { mise en }\end{array}$ & \multicolumn{4}{|c|}{ Intégration de ressources techniques pour créer des MMORPG sur Internet. } \\
\hline & Après & Internet & - & - & - \\
\hline \multirow{3}{*}{ Jémulex } & Avant & PC & + & - & + \\
\hline & $\begin{array}{c}\text { Stratégie } \\
\text { mise en } \\
\text { ouvre }\end{array}$ & \multicolumn{4}{|c|}{$\begin{array}{l}\text { Intégration des compétences liées à la distribution en utilisant Internet pour } \\
\text { diffuser ses jeux. }\end{array}$} \\
\hline & Après & Internet & - & - & - \\
\hline
\end{tabular}

Tableau 5 : Stratégies d'intégration misent en œuvre en fonction des sources de dépendance

Le tableau 5 synthétise les différentes sources de dépendance à l'origine du choix de la stratégie d'intégration mise en œuvre par les trois studios. Pour Jemulex, c'est avant tous sa position défavorable dans la filière qui l'a poussé à former ses salariés pour maîtriser la diffusion de son produit via la création de sites Internet. Dans le cas de MOP, maîtrisant déjà la partie aval de la filière, c'est principalement la nature contraignante du support technique qui l'a influencé à faire ce choix. Il a donc embauché des employés possédant des compétences techniques afin de se libérer des standards des fabricants. Enfin, le choix stratégique de Créajeux provient de son manque de ressources financières, mais surtout du support technique contraignant qu'il exploitait et de sa position défavorable dans la filière. Face à ces multiples sources de dépendance, il a embauché des ressources humaines et techniques pour se soustraire aux standards des fabricants et maîtriser la diffusion sur Internet.

\subsection{DISCUSSION}

Dans cette section, nous discutons les sources de dépendance, puis les stratégies mises en œuvre et enfin l'influence des sources sur la stratégie.

La taille, seule source mineure de dépendance : dans la littérature, la taille est souvent considérée comme une source importante de dépendance (Gardet et Mothe, 2008). Dans les cas étudiés, la taille n'a d'importance que lorsqu'il s'agit de la commercialisation de produits tangibles (consoles et PC). Le cas Flashgame coopérant avec un studio de plus grande taille illustre ce phénomène. Il est en situation d'interdépendance concurrentielle (Casciaro et Piskorski, 2005) et la différence de taille fait naître des asymétries de dépendance (Gulati et Stych, 2007). Toutefois, cela lui permet de diminuer sa dépendance symbiotique face à l'éditeur. Lorsque les studios développent des produits sur des marchés dématérialisés (internet), la taille n'a plus d'importance. En effet, pour Jemulex par exemple, l'utilisation d'Internet permet un accès direct au client final sans distributeur (Le Diberder, 2002). 
L'intégration plus courante que la coopération: les studios semblent privilégier l'intégration des ressources et compétences plutôt que la coopération (3 cas sur 5) - toute tentative de généralisation étant toutefois très difficile à effectuer sur un nombre de cas limité. L'intégration engendre des coûts supplémentaires (notamment financiers) et diminue la flexibilité des studios mais permet de conserver la liberté éditoriale. Cette liberté est extrêmement importante pour les dirigeants des studios vidéo car il s'agit de leur vocation et de leur cœur de métier: la création artistique. Ainsi, la motivation première des dirigeants pour minimiser la dépendance n'est pas tant l'aspect financier, contrairement aux industries non culturelles, mais précisément cette liberté créative (Storz, 2008). Dans la littérature, la mise en œuvre de coopération est souvent perçue comme un moyen pour accéder à de nouvelles ressources et compétences (Stieglitz et Heine, 2007), se focaliser sur le cœur de métier et réaliser des économies d'échelle (Powell, 1987). A contrario, l'intégration verticale est généralement associée à une rigidité de fonctionnement et à un manque de réactivité face à un environnement de plus en plus turbulent (Villalonga et McGahan, 2005). Pourtant, nos études de cas tendent à montrer que, dans l'industrie du jeu vidéo, la tendance est inversée. Trois principales raisons peuvent expliquer ce phénomène : (1) l'extrême hétérogénéité des cultures d'entreprises tend à augmenter les coûts de coordination, (2) la valeur ajoutée repose en grande partie sur la créativité des studios, qui est difficile à mesurer et à répartir lors d'une coopération et (3) lors d'une coopération, la liberté créative est diminuée car il faut s'accorder sur les objectifs poursuivis (Hamel, Doz et Prahalad, 1989).

\begin{tabular}{|c|c|c|c|c|c|c|}
\hline & \multicolumn{5}{|c|}{ Sources de dépendance } & \multirow{3}{*}{$\begin{array}{l}\text { Stratégies mises } \\
\text { en ouvre }\end{array}$} \\
\hline & \multicolumn{3}{|c|}{ Ressources et compétences } & \multirow{2}{*}{$\begin{array}{c}\text { Nature du } \\
\text { support } \\
\text { technologique }\end{array}$} & \multirow{2}{*}{$\begin{array}{l}\text { Position dans } \\
\text { la filière }\end{array}$} & \\
\hline & Financières & $\begin{array}{l}\text { Cession des } \\
\text { droits de PI } \\
\end{array}$ & $\begin{array}{c}\text { Absence de } \\
\text { réputation }\end{array}$ & & & \\
\hline Flashgame & +++ & +++ & +++ & +++ & +++ & $\begin{array}{l}\text { coopération } \\
\text { inéquitable }\end{array}$ \\
\hline Evolugame & ++ & +++ & ++ & +++ & +++ & $\begin{array}{c}\text { coopération } \\
\text { égalitaire }\end{array}$ \\
\hline Créajeux & ++ & - & - & +++ & ++ & $\begin{array}{c}\text { Intégration de } \\
\text { l'amont } \\
\text { (diversification } \\
\text { des supports) } \\
\end{array}$ \\
\hline Jemulex & + & - & - & - & + & $\begin{array}{c}\text { Intégration de } \\
\text { l'aval (intégration } \\
\text { de la distribution) } \\
\end{array}$ \\
\hline MOP & - & - & - & +++ & + & $\begin{array}{c}\text { Intégration de } \\
\text { l'amont }\end{array}$ \\
\hline & & $\begin{array}{r} \\
+++/++ \\
-: \text { sourc }\end{array}$ & $\begin{array}{l}\text { Légen } \\
\text { degré de dé } \\
\text { dépendanc }\end{array}$ & $\begin{array}{l}\text { : } \\
\text { ndance du stud } \\
\text { 'a pas d'influeı }\end{array}$ & & \\
\hline
\end{tabular}

Tableau 6 : Sources de dépendance influençant la stratégie mise en œuvre 
Trois principaux résultats sont mis en avant dans le tableau 6 :

- Aucun des studios étudiés ne maîtrise l'intégralité des ressources et compétences nécessaires au développement d'un jeu vidéo. Le manque de ressources et compétences en interne implique une dépendance des studios par rapport aux fabricants (qui détiennent les ressources techniques) et/ou par rapport aux éditeurs (qui possèdent les ressources financières). La théorie de la dépendance des ressources (Pfeffer et Salancik, 1978), s'avère utile pour faire le lien entre le type de ressource (ou compétence) recherchée par les studios et leur degré de dépendance vis à vis des autres acteurs de la filière. Deux formes d'interdépendance peuvent être identifiées : l'interdépendance symbiotique et l'interdépendance concurrentielle (Pfeffer et Nowak, 1976 ; Casciaro et Piskorski, 2005). Dans l'industrie du jeu vidéo, l'interdépendance symbiotique est davantage observée. En effet, les studios cherchent en priorité à sélectionner des acteurs reliés verticalement dans la filière. Toutefois, l'interdépendance concurrentielle est également observée, notamment dans les relations entre studios. Par exemple, pour augmenter leur pouvoir de négociation face aux autres acteurs de la filière, Flashgame et Evolugame coopèrent avec des concurrents. Les studios partenaires sont alors amenés à développer ensemble un jeu et se retrouvent en situation d'interdépendance concurrentielle ;

- Les deux studios ayant perdu leurs DPI (Flashgame et Evolugame) ont eu recours à la coopération afin de mutualiser leurs capacités de financement et d'obtenir un pouvoir de négociation supérieur face aux éditeurs. Ceci leur a permis de limiter l'investissement de l'éditeur afin de conserver les DPI et de diminuer leur degré de dépendance. $A$ contrario, dès lors que les DPI sont conservés, la stratégie privilégiée par les studios est l'acquisition de ressources et compétences amont (Créajeux et MOP) et/ou aval (Jémulex et Créajeux). Chesbrough et al. (2006) considèrent les DPI comme des actifs stratégiques devant être développés, vendus ou achetés. Or, dans deux cas (Flashgame et Evolugame), aucune des trois alternatives préconisées par Chesbrough et al. (2006) n'est mise en œuvre. En effet, ces deux studios choisissent la coopération afin de cocréer (depuis la genèse) un jeu et se partager les DPI. Dans une optique de complémentarité des ressources, cette volonté de partage des DPI fait référence à une volonté de maîtriser les ressources juridiques « objets ${ }^{24} »$ (Roquilly, 2006). Toutefois, il

\footnotetext{
${ }^{24}$ Roquilly (2006) distingue deux types de ressources juridiques. Les ressources « objets », qui représentent les contrats, les DPI, (etc.) et les ressources «connaissances", qui traduisent la connaissance des lois, des mécanismes juridiques, (etc.).
} 
est intéressant de souligner qu'aucun des deux studios n'avait une connaissance du statut juridique du jeu vidéo, ni même des lois ou des mécanismes juridiques à l'œuvre dans leur filière ;

- Flashgame et Evolugame ne bénéficient pas vraiment d'une réputation dans l'industrie. La réputation se construit à partir de deux principaux éléments (Rhee et Valdez, 2009) : des prestations de qualité et une visibilité face aux autres acteurs de la filière. Dans l'industrie du jeu vidéo, cette visibilité passe par la détention des DPI. La possession de ses droits augmente la liberté créative des studios et leur permet de dévoiler leur savoirfaire. Dans les industries manufacturières, il est possible d'être un sous-traitant réputé même si les DPI ne sont pas détenus en interne (Rhee et Valdez, 2009). En revanche, dans les industries culturelles, la détention des DPI confère une valeur intrinsèque au studio et contribue à sa réputation (Corbel, 2003).

\section{CONCLUSION}

L'analyse de la littérature a conduit à identifier les principales sources de dépendance et leur influence dans le processus du choix stratégique. Les cinq cas confortent l'influence de ces sources sur les stratégies instaurées par les studios de jeux vidéo. Trois principaux apports émergent de cette recherche :

- Le manque de ressources et compétences apparait comme étant la première source de dépendance des studios. En revanche, la taille ne semble pas avoir d'importance. Ainsi, le fait d'être un studio de petite taille ne signifie pas qu'il sera plus dépendant des autres acteurs qu'un studio de grande taille. D'autres dimensions, telles que la possession des DPI ou une forte réputation, permettent à un studio de petite taille d'être peu dépendant des autres acteurs ;

- La majorité des recherches analysent l'influence des choix stratégiques sur la performance de l'entreprise (Wang et Zajac, 2007). Ici, l'objectif n'était pas de savoir si la coopération était plus favorable que l'intégration, mais plutôt d'analyser les sources pouvant avoir un impact sur ce choix. En effet, même si la coopération est souvent perçue comme plus favorable, elle ne semble pas toujours privilégiée par les studios car elle implique le partage des ressources créatives (Storz, 2008) et de la liberté créative. De plus, les spécificités culturelles (langage, organisation du travail, etc.) des studios entraînent souvent des conflits lors de coopérations (Gil et Spiller, 2007) et augmentent les coûts de coordination. Les études empiriques qui analysent le degré de dépendance 
entre acteurs sont très rarement réalisées au sein d'industries culturelles. A contrario, les relations entre fournisseurs et donneurs d'ordres dans l'industrie automobile font l'objet d'un grand nombre de recherches (Perrow, 1970 ; Tinlot et Mothe, 2005). Pourtant, les spécificités des industries culturelles ne permettent pas de transférer les résultats d'une autre industrie, et ces spécificités méritent d'être étudiées ;

- Enfin, une hiérarchisation des sources de dépendance semble influencer le choix des stratégies des studios. Pour un studio comme Flashgame ou Evolugame, soumis à l'influence de toutes les sources de dépendance, la stratégie choisie vise d'abord à obtenir les DPI et à se construire une réputation. Pour un studio parvenu à conserver ses droits et à être reconnu, la stratégie retenue semble être l'intégration des ressources et compétences de l'amont afin de diminuer la dépendance technologique (Créajeux et MOP). Une fois la dépendance technologique contournée (Jemulex), l'intégration de ressources de l'aval semble privilégiée. Ainsi, la diminution du degré de dépendance semble passer par une succession d'étapes depuis la détention des DPI jusqu'à l'intégration des compétences de l'aval. Ce processus évoque la notion de dépendance de sentier (Storz, 2008) : dans certaines industries, les entreprises sont soumises à une même trajectoire d'évolution.

Des travaux ultérieurs pourraient pallier les limites de la présente recherche, notamment en introduisant d'autres facteurs contextuels comme l'âge du studio ou sa nationalité. L'analyse se focalisant sur un type d'acteur au sein de l'industrie du jeu vidéo, les studios, il serait intéressant d'observer les sources de dépendance des autres acteurs de la filière afin de proposer une analyse des interdépendances. Cette analyse du niveau de dépendance mutuelle (Casciaro et Piskorski, 2005) permettrait d'affiner notre compréhension des stratégies mises en œuvre par les studios. Enfin, les studios étudiés sont des entreprises de taille moyenne voire petite. Le rôle du dirigeant dans ces structures est donc fondamental (Chollet et Géraudel, 2010). Il est probable que la personnalité du dirigeant influence fortement les choix stratégiques effectués. Un dirigeant ayant un fort degré d'aversion au risque privilégiera probablement la sécurité de la relation asymétrique au détriment d'une liberté éditoriale. Dans les cas étudiés, les dirigeants avaient la volonté de faire croître leur entreprise et ont systématiquement cherché à minimiser leur degré de dépendance. Toutefois, certains studios, non étudiés dans cette étude, privilégient la sécurité. 


\section{BIBLIOGRAPHIE}

Arrègle J.L. (2006), “Analyse« Resource Based » et identification des actifs stratégiques”, Revue Française de Gestion, 1(160), 241-259.

Balakrishnan S., Koza M.P. (1993), "Information Asymmetry, Adverse Selection, and Joint Ventures", Journal of Economic Behavior and Organization, 20, 99-117.

Barney J.B. et Hansen M.H. (1994), "Trustworthiness as a Source of Competitive Advantage", Strategic Management Journal, 15, 175-190.

Blau P. (1964), Exchange and Power in Social Life, New York: Wiley.

Brousseau E. (1993), L'économie des contrats, technologies de l'information et coordination interentreprises, Paris : PUF.

Busija E.C., O’Neill H.M., Zeithaml C.P. (1997), "Diversification Strategy, Entry Mode, and Performance: Evidence of Choice and Constraints", Strategic Management Journal, 18(4), 321-327.

Busson A., Evrard Y. (1987), Portraits économiques de la culture, La documentation française, Paris.

Casciaro T., Piskorski M.J. (2005), "Power Imbalance Mutual Dependence and Constraint Absorption: A Closer look at Resource Dependence Theory", Administrative Science Quarterly, 50, 167-199.

Castaneda M.A. (2006), "The Hold-up Problem in a Repeated Relationship", International Journal of Industrial Organization, 24(5), 953-970.

Chesbrough H. (2003a), Open Innovation : The New Imperative for Creating and Profiting from Technology, MA: Harvard Business School Press, Boston.

Chesbrough H. (2003b), "The Logic of Open Innovation: Managing Intellectual Property", California Management Review, 45(3), 33-58.

Chesbrough H., Vanhaverbeke W., West J. (2006), Open Innovation: Researching a New Paradigm, Oxford: Oxford University Press.

Chollet B., Géraudel M. (2010), "Réseau personnel, personnalité du dirigeant de PME et accès aux informations sur les marchés", Finance Contrôle Stratégie, Forthcoming.

Cohendet P., Simon L. (2007), "Playing across the playground: paradoxes of knowledge creation in the videogame firm", Journal of Organizational Behavior, 28(5), 587-605.

Conner K.R., Prahalad C.K. (1996), “A Resource-Based Theory of the Firm: Knowledge versus Opportunism”, Organization Science, 7(5), 477-501.

Cook K.S. (1977), "Exchange and Power in Networks of Interorganizational Relations", Sociological Quarterly, $18(1), 62-82$.

Corbel V. (2003), Management stratégique des droits de la propriété intellectuelle, Gualino Editeur, Paris.

Dahl R.A. (1957), “The Concept of Power”, Behavioral Sciences, 2, 201-215.

Das T.K., Teng B.S. (1998), "Between Trust and Control: Developing Confidence in Partner Cooperation in Alliances", Academy of Management Review, 23(3), 491-512.

Dibiaggio L. (1999), "Apprentissage, coordination et organisation de l'industrie une perspective cognitive", Revue d'Economie Industrielle, Vol. 88, p. 111-136.

Dyer J.H. (1997), "Effective Interfirm Collaboration: How Firms Minimize Transaction Costs and Maximize Transaction Value", Strategic Management Journal, Vol. 18, nº7, p. 535-556.

Emerson R.M. (1962), "Power-Dependence Relations", American Sociological Review, 27(1), 31-41.

Fonrouge C. (2007), "Relations externes et innovation : le cas du secteur des biotechnologies", Revue Française de Gestion, 170, 117-133.

Gardet E., Mothe C. (2008), "The influence of hub firm size and degree of dependence on the coordination mechanisms applied to innovation networks", EGOS colloquium, Vrije University Amsterdam, Pays-Bas.

Gil R., Spiller P. (2007), “The Organizational Dimensions of Creativity: Motion Picture Production”, California Management Review, 50(1), 243-260.

Gulati R., Stych M. (2007), "Dependence Asymmetry and Joint Dependence in Interorganizational Relationships: Effects of Embeddedness on a Manufacturer's Performance in Procurement Relationships", Administrative Science Quarterly, 52, 32-69.

Hagedoorn J. (1993), "Understanding the Rationale of Strategic Technology Partnering. Interorganizational Modes of Cooperation and Sectoral Differences", Strategic Management Journal, 14(3), 371-385.

Hamel G., Doz Y.L. et Prahalad C.K. (1989), "Collaborate with your Competitor and Win”, Harward Business Review, 67(1), 133-139.

Hamel G. et Prahalad C.K. (1994), Competing for the future, Harvard Business School Press.

Hennart J.F. (1988), “A Transaction Costs Theory of Equity Joint Ventures”, Strategic Management Journal, Vol. 9, p. 361-374.

Herman L. (1999), Phoenix. The Rise and Fall of Video-Games, (Second edition), Rolenta Press.

Ingham M. (1994). "L'apprentissage organisationnel dans les coopérations", Revue Française de Gestion, janvier-février, 105-119. 
Kinder M. (1991), Playing with Power in Movies, Television and Video Games, Oxford: University of California Press.

Kogut B., Singh H. (1988), "The Effect of National Culture on the Choice of Entry Mode", Journal of International Business Studies, fall, 411-432.

Klein B., Crawford R., Alchian A. (1978), "Vertical Integration Appropriable Quasi-rents and the Competitive Contracting Process", Journal of Law and Economics, 21(10), 297-326.

Koenig G. (1990), Management Stratégique, Paris, Nathan.

Kumar K., Van Dissel H.G. (1996), "Sustainable Collaboration: Managing Conflict and Cooperation in Interorganizational Systems", MIS Quarterly, 20(3), 279-300.

Lavie D. (2006), "The Competitive Advantage of Interconnected Firms: An Extension of the Resource-Based View", Academy of Management Review, 31(3), 638-658.

Le Diberder A. (2002), "La création de jeux vidéo en France en 2001", Étude du ministère de la culture et de la communication, Paris.

Liotard I. (1999), "Les droits de propriété intellectuelle, une nouvelle arme stratégique des firmes", Revue d'économie industrielle, 89(1), 69-84.

Miles M.B., Huberman M. (2003), Analyse des données qualitatives, $2^{\mathrm{e}}$ Edition, De Boeck.

Mohr J., Spekman R. (1994), "Characteristics of Partnership Success: Partnership Attributes, Communication Behavior, and Conflict Resolution Techniques", Strategic Management Journal, 15(2), 135-152.

Nooteboom B., De Jong G., Vossen R.W., Helper S., Sako M. (2000), "Network Interactions and Mutual Dependence: A Test in the Car Industry", Industry and Innovation, 7(1), 117-144.

Oliver C. (1990), "Determinants of Interorganizational Relationships: Integration and Future Directions", Academy of Management Review, 15(2), 241-265.

Parmentier G., Mangematin V. (2009), "Innovation et création dans le jeu vidéo. Comment concilier exploration et exploitation?", Revue Française de Gestion, 35(191), 77-87.

Perrow C. (1970), Organizational Analysis: A Sociological View, London: Tavistock.

Pfeffer J., Nowak P. (1976), "Joint Ventures and Interorganizational Interdependence", Administrative Science Quarterly, 21, 398-418.

Pfeffer J., Salancik G. (1978), The External Control of Organizations. A Ressource Dependence Perspective, New York: Harper \& Row.

Pisano G.P., Teece D.F. (2007), "How to Capture Value from Innovation: Shaping Intellectual Property and Industry Architecture", California Management Review, 50(1), 278-296.

Porter M.E. (1985), Competitive Advantage: Creating and Sustaining Superior Performance, New York: Free Press.

Powell W.W. (1987), “Hybrid Organizational Arrangements: New Form or Transitional Development?", California Management Review, 30(1), 67-87.

Rhee M., Valdez M.E. (2009), “Contextual Factors Surrounding Reputation Damage with Potential Implications for Reputation Repair", Academy of Management Review, 34(1), 146-168.

Rouet F. (2009), La création dans l'industrie du jeu vidéo, Etude du ministère de la culture et de la communication, Paris.

Schelling T. (1956), “An essay on bargaining”, American Economic Review, 46, 281-306.

Segrestin B. (2006), Innovation et coopération interentreprises comment gérer les partenariats d'exploration ? CNRS éd. (CNRS économie).

Stieglitz N., Heine K. (2007), "Innovations and the Role of Complementarities in a Strategic Theory of the Firm", Strategic Management Journal, 28, 1-15.

Storz C. (2008), "Dynamics in Innovation Systems: Evidence from Japan's Game Software Industry”, Research Policy, 37(9), 1480-1491.

Tinlot G., Mothe C. (2005), “Alliance asymétrique et pouvoir de négociation des partenaires : une relecture de l'alliance Matra-Renault”, Management International, 10(1), 31-49.

Toledano J. (1978), "A propos des filières industrielles", Revue d'Economie Industrielle, 6(6), 149-158.

Villalonga B., McGahan A.M. (2005), “The Choice among Acquisitions, Alliances and Divestitures", Strategic Management Journal, 26(13), 1183-1208.

Wang L. et Zajac E. (2007), “Alliance or Acquisition ? A Dyadic Perspective on Interfirm Resource Combinations”, Strategic Management Journal, 28(13), 1291-1317.

Warnier V., Lecocq X. et Demil B. (2006), "Le Business Model, un outil d'analyse", L'Expansion Management Review, 123, 96-109.

Williamson O.E. (1985), The Economic Institutions of Capitalism, The Free Press, New York.

Zaheer A., McEvily B. et Perrone V. (1998), "Does Trust Matter? Exploring the Effects of Interorganizational and Interpersonal Trust on Performance", Organization Science, 9(2), 141-159. 


\begin{tabular}{|c|c|c|c|c|c|c|c|}
\hline \multirow[b]{2}{*}{ 象 } & \multirow[b]{2}{*}{ Verbatim } & \multicolumn{4}{|c|}{ Sources de dépendance } & \multicolumn{2}{|c|}{ Stratégie } \\
\hline & & 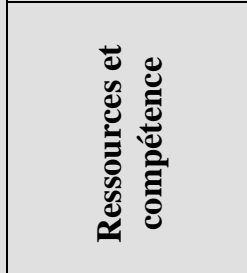 & 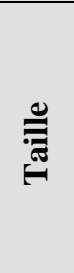 & 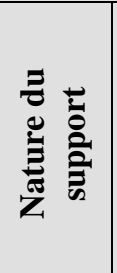 & 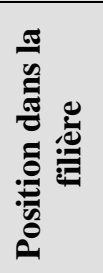 & 产 & ठี \\
\hline Flashgame & $\begin{array}{c}\text { «Pour nous l'éditeur c'est un passage obligé. On a pas le financement } \\
\text { en interne pour assurer tout le développement du jeu donc on fait appel } \\
\text { aux éditeurs. Bien sûr, en contrepartie il faut céder les droits. » } \\
\text { (Directeur commercial - Flashgame) }\end{array}$ & $\begin{array}{l}\text { Financières } \\
\text { et techniques } \\
\text { (DPI) }\end{array}$ & & & & & \\
\hline Evolugame & $\begin{array}{l}\text { «Notre réputation dans le milieu compte beaucoup pour nous, c'est } \\
\text { notre carte de visite, on décroche plus de projets ou on nous fait plus } \\
\text { confiance; mais ce n'est pas pour autant qu'on est totalement libre de } \\
\text { faire ce qu'on veut » (Directeur technique - Evolugame) }\end{array}$ & réputation & & & & & $\mathrm{X}$ \\
\hline Créajeux & $\begin{array}{c}\text { «On voulait essayer de se diversifier au maximum pour prolonger les } \\
\text { expériences liées à nos jeux. En plus, on n'était plus obligé d'utiliser la } \\
\text { technologie des fabricants : donc c'était vraiment un avantage pour } \\
\text { nous »(Responsable R\&D - Créajeux) }\end{array}$ & $\begin{array}{l}\text { Humaines et } \\
\text { techniques }\end{array}$ & & $\mathrm{X}$ & & & \\
\hline Jemulex & $\begin{array}{c}\text { «Notre petite taille n'est pas un handicap, on tient à rester petit pour } \\
\text { etre flexible et d'ailleurs on n'a de compte à rendre à personne sauf aux } \\
\text { distributeurs »(Directeur du studio - Jémulex) }\end{array}$ & & $X$ & & & & \\
\hline MOP & $\begin{array}{c}\text { «Depuis le début on privilégie l'autonomie, on essaye de tous faire nous } \\
\text { même, c'est plus simple et on fait ce qu'on veut. Si on a pas de quoi le } \\
\text { faire, on l'internalise. »(Responsable projet - MOP) }\end{array}$ & & & & & $\mathrm{X}$ & \\
\hline
\end{tabular}

